\title{
Investigações brasileiras sobre o transtorno de personalidade borderline: uma revisão integrativa
}

\section{Investigations on the brazilian borderline personality disorder: an integrative review}

\author{
Lucas de Holanda Leite ${ }^{1}$. Eugenio de Moura Campos². \\ 1 Médico, discente do curso de Residência em Psiquiatria do Hospital Universitário Walter Cantídio, Fortaleza, Ceará, Brasil. 2 \\ Doutor em Farmacologia, professor adjunto da Universidade Federal do Ceará (UFC) e Universidade de Fortaleza (UNIFOR), \\ Fortaleza, Ceará, Brasil.
}

\section{RESUMO}

Transtorno de Personalidade Borderline (TPB) é um sofrimento mental grave que apresenta demandas complexas à atenção clínica e à sociedade, principalmente devido à instabilidade das relações interpessoais e intrapessoais, assim como a impulsividade acentuada e o risco de suicídio. O presente estudo objetivou realizar uma análise da produção científica brasileira acerca do TPB. Trata-se de uma revisão integrativa da literatura publicada no período de janeiro de 2000 a dezembro de 2014 nas bases de dados: Lilacs e Medline. A seleção foi baseada no entre cruzamento de palavras-chave indexadas nos Descritores em Ciências da Saúde: "Borderline Personality Disorder", "Therapy", "Psychotherapy" e "Drug Therapy", sendo utilizadas todas as combinações possíveis, com os recursos disponibilizados pelos operadores booleanos. A partir desse levantamento, identificou-se a ênfase dada à busca de diagnóstico por neuroimagem; o relato de experiências exitosas de psicoterapias e a lacuna em pesquisas que discutam a utilização de fármacos. Evidencia-se a complexidade dos critérios diagnósticos e as graves características do TPB, como autolesões e tentativas de suicídio. Por fim, ressalta-se a necessidade de que se construa uma atenção em saúde integral, envolvendo familiares e comunidade, na busca por produzir um cuidado resolutivo.

Palavras-chave: Transtorno de personalidade borderline. Psiquiatria. Psicopatologia.

\section{ABSTRACT}

The Borderline Personality Disorder (BPD) is a serious mental disorder that presents complex demands of clinical care and society, mainly due to the instability of interpersonal and intrapersonal relationships, as well as marked impulsivity and suicide risk. This study aimed to carry out an analysis of the scientific production about the TPB. This is an integrative review of the literature published from January 2000 to December 2014 in the databases Lilacs and Medline. The selection was based on between intersection of keywords indexed in Descriptors Health Sciences: "Borderline Personality Disorder", "Therapy", "Psychotherapy" and "Drug Therapy", being used all possible combinations, with the resources deployed by Boolean operators. From this survey, it identified the emphasis on the search of diagnostic neuroimaging; the account of successful experiences of psychotherapy and the gap in research that discuss the use of drugs. The complexity is evident in the diagnostic criteria and the serious TPB characteristics such as self-injury and suicide attempts. Finally, it emphasizes the need to build a full health care, involving family and community, seeking to produce a terminating care.

Keywords: Borderline personality disorder. Psychiatry. Psychopathology.

Autor correspondente: Lucas de Holanda Leite, Rua Capitão Francisco Pedro, 1290, Rodolfo Teófilo, Fortaleza, Ceará. Telefone: +55 85 33668149. E-mail: drlucasleite@hotmail.com

Conflito de interesses: Não há qualquer conflito de interesses por parte de qualquer um dos autores.

Recebido em: 13 Set 2015; Revisado em: 24 Fev 2016; Aceito em: 24 Fev 2016. 


\section{INTRODUÇÃO}

O Transtorno de Personalidade Borderline (TPB) é uma categoria de sofrimento mental que apresenta demandas complexas à atenção clínica e à sociedade. A sua sintomatologia é descrita pela Associação Americana de Psiquiatria por meio do Manual Diagnóstico e Estatístico de Transtornos Mentais V (DSM-V), como apresentando um padrão de instabilidade das relações interpessoais e intrapessoais (no tocante à autoimagem do sujeito, ou seja, à percepção de si), além da presença de impulsividade acentuada. Assim, os seus sintomas convergem para estados disfuncionais, podendo comprometer o bom relacionamento social, principalmente, no âmbito dos afetos. $^{1}$

O DSM-5 indica que os primeiros quadros sintomatológicos do TPB surgem no começo da idade adulta e podem aparecer de modo súbito, além de, muitas vezes, caótico e intenso. ${ }^{1}$ Destarte não existe um padrão e/ou uma situação de gatilho específico, pois o seu aparecimento se dá em variados e diferentes contextos. Dessa forma, compreende-se o seu caráter complexo e desafiador que envolve os saberes e as práticas da Psiquiatria e da Saúde Mental.

O entendimento diagnóstico atual acerca do TPB foi formulado desde a década de 1980 pelo DSM-III. Antes disso, esse quadro sintomatológico era descrito como um estado intermediário entre neurose-psicose, e não como um transtorno de personalidade específico. Depois disso, a sua situação nosotáxica e nosológica passou a ser mais bem estudada, passando do espectro da esquizofrenia para o capítulo dos transtornos da personalidade. Destaca-se, ainda, que essa categoria passou a agrupar dois subtipos: o tipo impulsivo e o tipo borderline, sendo a este último o tipo que o presente estudo dá maior ênfase. ${ }^{2}$

Essa conceituação supracitada foi importante para definir uma classificação específica do TPB e, assim, nortear a clínica e os processos terapêuticos que a envolvem. Ressalta-se, ainda, que o conceito passou por algumas reformulações e hoje engloba alguns termos igualmente definidores, tais como: limítrofe (designando o estado do sujeito que está no "limite" da sanidade); e borderline (fazendo referência à situação de fronteira, na "borda" das dimensões neurótica e psicótica).

Essas características diagnósticas foram formuladas a partir de diferentes estudos, principalmente os realizados pelo psiquiatra Robert Spitzer (considerado por alguns como o pai da psiquiatria moderna) e seus colegas. Em uma das pesquisas realizadas pelo grupo de Spitzer, foi demonstrada uma lista de critérios diagnósticos do comportamento borderline que se mostraram amplamente aplicáveis ao cotidiano da prática psiquiátrica. A partir disso, as teorias modernas passaram a discorrer sobre os fatores genéticos inespecíficos que envolvem o TPB, bem como sobre os problemas do desenvolvimento associados com a negligência emocional e/ou abuso na infância. ${ }^{3}$ Reforçou-se, assim, a existência de pesquisas sobre a compreensão e análise neurobiológica, bem como o aperfeiçoamento de tratamentos e formas de manejo clínico do TPB.
Destaca-se que o DSM-5 (oficialmente publicado em 18 de maio de 2013), é a edição mais recente do Manual, sendo resultado de um processo de doze anos de estudos, revisões e pesquisas científicas. Essa reformulação teve como objetivo trazer maior segurança e embasamento científico atualizado para os profissionais da área de Psiquiatria. Diante disso, observa-se que o DSM IV (lançado em 1994) traçou uma descrição do TPB bastante significativa, apontando os seus principais sintomas e características, bem como algumas das possíveis estratégias de tratamento. Com o DSM-5, identificam-se algumas mudanças e acréscimos de alguns transtornos no manual. ${ }^{4}$

No entanto, no que se refere aos Transtornos de Personalidade - dentre os quais se insere o TPB -, apreende-se que os critérios diagnósticos não sofreram mudanças em relação aos apresentados no DSM-IV. O novo manual continua reunindo os mesmos transtornos divididos em três grupos: Grupo A - Transtornos de Personalidade Paranóide, Esquizóide e Esquizotípica; Grupo B - Transtornos de Personalidade Anti-Social, Borderline, Histriônica e Narcisista; e Grupo C - Transtornos de Personalidade Esquiva, Dependente e Obsessivo-Compulsiva, apresentando as mesmas características diagnósticas, as quais foram citadas

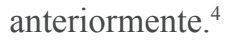

Outro importante compêndio diagnóstico é o formulado pela "Classificação Internacional de Doenças e Problemas Relacionados à Saúde" (CID-10, 1993), que define Borderline na categoria referente aos Transtornos de Personalidade com Instabilidade Emocional (F60.3). Assim, tem-se a descrição como tipo borderline (limítrofe) e distinguem-se as seguintes características: comportamentos de instabilidade emocional e na autoimagem, assim como no tocante às preferências internas (incluindo a sexual), que estão, com frequência, pouco claras ou perturbadas.

A CID-10 aponta que, em geral, as pessoas com TPB apresentam sentimentos crônicos de vazio e uma propensão a se envolver em relacionamentos intensos e instáveis, o que potencializa a reincidência de crises emocionais. Outros comportamentos descritos são: esforço excessivo para evitar o abandono e ameaça/tentativa de suicídio, assim como ações de autolesão. Enfatiza-se, ainda, que existe tendência nítida a agir de modo imprevisível, sem consideração pelas consequências; humor imprevisível e caprichoso; tendência à agressividade e uma incapacidade de controlar os comportamentos impulsivos; tendência a adotar um comportamento de conflito com os outros, particularmente quando os atos impulsivos são contrariados ou censurados.

Destarte, compreende-se que os indivíduos com características TPB são severamente disfuncionais e, apesar de avaliarem adequadamente a realidade, apresentam comportamento inapropriado e incoerente com essa avaliação, o que dificulta o seu relacionamento em sociedade, potencialmente provocando conflitos. 
Observa-se que a prevalência média do TPB na população é estimada em $1,6 \%$, podendo chegar a $5,9 \%$. Destaca-se que essa prevalência é de aproximadamente $6 \%$ em contextos de atenção primária, de cerca de $10 \%$ entre os usuários ambulatoriais e de consultórios psiquiátricos e de mais ou menos $20 \%$ em indivíduos internados em leitos psiquiátricos. Salienta-se, ainda, que a sua incidência é maior no sexo feminino e que a sua prevalência tende a diminuir nas faixas etárias mais altas. ${ }^{1}$

Essa prevalência indica, portanto, a necessidade de que se desenvolvam estratégias de cuidado mais eficientes, pois, devido às características disfuncionais inerentes ao TPB, os indivíduos podem apresentar maiores dificuldades em se manter e apresentar resultados positivos no processo terapêutico.

No que se refere ao tratamento farmacológico são utilizados diferentes tipos de medicamentos e que seus efeitos são inconsistentes. ${ }^{3}$ Diante disso, evidencia-se, mais uma vez, a necessidade de que se invista em pesquisas de aprimoramento desses medicamentos, pois, apesar de algumas vezes apresentarem resultados, estes ainda são modestos e não se pode desconsiderar a probabilidade de abuso e os efeitos colaterais. Assim, enquanto não se concretizar um fármaco eficiente e confiável, deve-se prescrever com muita cautela e acompanhar os efeitos do uso com atenção.

Diante disso, o presente trabalho tem por objetivo a análise dessa produção científica brasileira, a partir de uma revisão integrativa acerca das investigações realizadas sobre o TPB, no tocante às suas características diagnósticas e ao seu manejo clínico-terapêutico.

\section{MÉTODO}

Trata-se de uma revisão integrativa da literatura brasileira publicada sobre o TPB. Essa metodologia permitiu a análise de pesquisas relevantes sobre o tema e seus resultados potencialmente contribuirão para a tomada de decisão e a melhoria da prática clínica em Psiquiatria.

Essa revisão integrativa consistiu na síntese do estado do conhecimento acerca do cuidado destinado aos sujeitos diagnosticados com o TPB, sintetizando os múltiplos estudos publicados, trazendo conclusões gerais a respeito do assunto, além de apontar lacunas do conhecimento científico nessa seara.

Para a elaboração desta pesquisa seguiram-se as seis etapas apresentadas: reflexão e consolidação da pergunta norteadora; seleção e busca dos artigos (por meio de estratégias de refinamento dos achados - critérios de inclusão e exclusão); definição das informações a serem extraídas dos estudos selecionados; avaliação crítica dos trabalhos selecionados; discussão dos resultados e apresentação da revisão integrativa. ${ }^{5}$

Para a primeira etapa foi elaborada a seguinte questão norteadora para o estudo: "Quais as evidências científicas brasileiras acerca das características diagnósticas e do manejo clínico-terapêutico do Transtorno de Personalidade Borderline?".

Na etapa seguinte foi feita a seleção dos trabalhos, por meio de busca das publicações da literatura científica, no período de Janeiro 2000 a dezembro de 2014, nos idiomas inglês, espanhol e português, tendo o Brasil como país de filiação, estando disponível em formato de artigo, indexado na Biblioteca Virtual em Saúde (BVS), especificamente nas seguintes bases de dados científicos: LILACS (Literatura Latino-Americana e do Caribe em Ciências da Saúde) e MEDLINE (Literatura Internacional em Ciências da Saúde) - que foram as únicas que apresentaram produções que se adequaram aos critérios de refinamento da busca.

Dessa forma, os critérios de inclusão estabelecidos para a seleção dos artigos foram: ser artigo original; apresentar resposta temática à pergunta investigativa; ter disponibilidade eletrônica na forma de texto completo; ter sido publicado no período supracitado; apresentar versão em idioma inglês, espanhol e/ou português; e possuir o Brasil como país de filiação. Foram excluídos: estudos repetidos e os que, apesar de apresentarem todos os critérios de inclusão, davam maior ênfase a um tema diferente do investigado.

Para a busca dos artigos, foram utilizadas três palavras chaves indexadas nos Descritores em Ciências da Saúde (DeCS): "Borderline Personality Disorder" (Transtorno de Personalidade Borderline), “Therapy" (Terapia), "Psychotherapy" (Psicoterapia) e "Drug Therapy" (terapia farmacológica), sendo utilizadas todas as combinações possíveis, com os recursos disponibilizados pelos operadores booleanos AND e OR.

Para uma melhor análise crítica dos estudos pré-selecionados, foram elaboradas categorias de destaque nas produções analisadas, enfatizando, assim, os principais assuntos abordados nos artigos.

Por fim, seguiram-se leituras repetidas e elaboração de fichamentos dos trabalhos selecionados, na busca por concretizar uma síntese geral e conclusiva das produções científicas publicadas nos últimos anos no Brasil, que abordam as das investigações realizadas sobre o TPB, no tocante às suas características diagnósticas e ao seu manejo clínicoterapêutico.

\section{RESULTADOS}

Realizada a busca na Biblioteca Virtual em Saúde (BVS) - com todas as combinações possíveis dos descritores: "Borderline Personality Disorder", "Therapy", "Psychotherapy" e "Drug Therapy" -, foram encontradas 2.821 produções científicas. Após a adoção dos filtros - formato artigo, disponível em texto completo, e Brasil como país de filiação -, foram selecionados 27 artigos. Então, seguiu-se à pré-seleção, por meio da leitura dos títulos e dos resumos, configurando-se em uma préanálise do material. Ao término dessa fase, foram escolhidos os 15 artigos que respondiam à pergunta: "Quais as evidências científicas brasileiras acerca das características diagnósticas e 
do manejo clínico-terapêutico do Transtorno de Personalidade Borderline?".

Os 12 artigos que foram excluídos apresentaram temas que não respondiam ao objetivo do presente artigo. Um desses trabalhos foi excluído da seleção por apresentar como tema central a análise acerca do papel molecular e usos clínicos nos transtornos psiquiátricos dos ácidos graxos, sem especificamente tratar do TPB. Outro artigo que também apresentou tema central distinto intitula-se "Disfunção erétil e psicopatologia: um estudo clínico", e traz o TPB apenas como caracterização de um dos sujeitos investigados. Essa caracterização também é feita no trabalho "A relação entre transtornos mentais e tipos de crime em detentos em uma prisão brasileira", que também foi excluído por não responder ao objetivo da presente análise. Por motivo semelhante, foi retirado da seleção o estudo "A prevalência de transtornos mentais em presos na cidade de Salvador, Bahia, Brasil”. Alguns trabalhos foram excluídos, por sua vez, por enfatizarem teorias e práticas clínicas de saúde mental, mas sem se referirem especificamente ao TPB, quais sejam: "Psicanálise e Universidade: a interface possível por meio da pesquisa psicanalítica clínica/Alice quebra-vidros"; "Verdade como uma forma de desenvolver e preservar o espaço para pensar na mente do paciente e analista"; e "Tratamento farmacológico da impulsividade e do comportamento agressivo". Outros estudos foram excluídos por trazer o TPB apenas como um dos exemplos de comorbidades psiquiátricas, quais sejam: "Relação entre neuropsicológica e aspectos clínicos e tentativas de suicídio em pacientes bipolares"; "O transtorno bipolar e transtornos psiquiátricos e médicos simultâneos"; "O polimorfismo do transportador de serotonina (5-HTTLPR) um marcador potencial de comportamento suicida em pacientes com transtorno bipolar?"; e "Tentativa de suicídio em mulheres em idade reprodutiva". Outro estudo, intitulado "Alterações corticais morfologia em mulheres com transtorno de personalidade borderline: uma abordagem multimodal" se apresentou repetido nas duas bases dados (LILACS E MEDLINE). Os artigos incluídos nesse estudo estão dispostos no Quadro 1.

Quadro 1. Apresentação da síntese dos trabalhos incluídos na revisão integrativa.

\begin{tabular}{|c|c|c|c|c|}
\hline $\mathbf{N}^{0}$ & Título & $\begin{array}{c}\text { Periódico e Base de } \\
\text { Dados }\end{array}$ & Autoria & Principal Temática \\
\hline 01 & $\begin{array}{l}\text { Alterações na morfologia cortical } \\
\text { em mulheres com transtorno de } \\
\text { personalidade borderline: uma } \\
\text { abordagem multimodal }\end{array}$ & $\begin{array}{l}\text { Rev Bras Psiquiatr } \\
\text { LILACS/ MEDLINE }\end{array}$ & $\begin{array}{l}\text { Araujo TB, Araújo GM Filho, } \\
\text { Sato JR, Araújo CM, Lisondo } \\
\text { CM, Carret H Júnior, et al }{ }^{4} \\
2014 .\end{array}$ & $\begin{array}{l}\text { Compreensão adequada da } \\
\text { neurobiologia do TPB pode } \\
\text { ajudar a fornecer a base para } \\
\text { intervenções mais precoces e } \\
\text { eficazes. }\end{array}$ \\
\hline 02 & $\begin{array}{l}\text { Amygdalar volume in borderline } \\
\text { personality disorder with and without } \\
\text { comorbid post-traumatic stress } \\
\text { disorder: a meta-analysis }\end{array}$ & $\begin{array}{l}\text { CNS Spectr } \\
\text { MEDLINE }\end{array}$ & $\begin{array}{l}\text { Almeida CP, } \\
\text { A, Carvalho CS, Penzel } \\
\text { VB, Araújo-Neto C, Quarantini } \\
\text { LC, et al }{ }^{11} \\
2012 .\end{array}$ & $\begin{array}{l}\text { Estudos descobriram um } \\
\text { volume da amígdala menor em } \\
\text { pacientes com TPB em relação } \\
\text { aos demais sujeitos. Trata-se } \\
\text { de uma meta-análise sobre o } \\
\text { assunto. }\end{array}$ \\
\hline 03 & $\begin{array}{l}\text { Comorbidade entre bulimia e } \\
\text { transtorno de personalidade borderline: } \\
\text { implicações para o tratamento }\end{array}$ & $\begin{array}{l}\text { Rev. latinoam. } \\
\text { psicopatol. Fundam } \\
\text { LILACS }\end{array}$ & $\begin{array}{c}\text { Rosa BP, Santos } \mathrm{MA}^{15} \\
2011 .\end{array}$ & $\begin{array}{l}\text { O estudo investiga o manejo } \\
\text { clínico de pacientes com } \\
\text { comorbidade entre transtorno } \\
\text { alimentar e transtorno de } \\
\text { personalidade borderline. }\end{array}$ \\
\hline 04 & $\begin{array}{l}\text { Ensino da psicoterapia no atendimento } \\
\text { psiquiátrico dos pacientes com } \\
\text { transtorno de personalidade borderline }\end{array}$ & $\begin{array}{l}\text { Psicol. teor. Pesqui } \\
\text { LILACS }\end{array}$ & $\begin{array}{l}\text { Vidal M, Lowenkron } \mathrm{T}^{2} . \\
2010 .\end{array}$ & $\begin{array}{l}\text { Kernberg sobre uma abordagem } \\
\text { psicoterápica do paciente com } \\
\text { TPB. }\end{array}$ \\
\hline 05 & $\begin{array}{l}\text { Adolescência e organização } \\
\text { de personalidade } \\
\text { caracterização dos vínculos afetivos }\end{array}$ & $\begin{array}{l}\text { Paidéia (Ribeirão } \\
\text { Preto) } \\
\text { LILACS }\end{array}$ & $\begin{array}{c}\text { Jordão AB, Ramires } V^{8} \\
\text { 2010a. }\end{array}$ & $\begin{array}{l}\text { Revisão abrangente e não- } \\
\text { sistemática da literatura sobre: } \\
\text { adolescentes - laços afetivos } \\
\text { e transtorno de personalidade } \\
\text { borderline. }\end{array}$ \\
\hline 06 & $\begin{array}{l}\text { Vínculos afetivos de adolescentes } \\
\text { limítrofe e seus pais }\end{array}$ & $\begin{array}{l}\text { Rev. Psic.: Teor e } \\
\text { pesq } \\
\text { LILACS }\end{array}$ & $\begin{array}{c}\text { Jordão AB, Ramires VR }{ }^{9} \\
\text { 2010b. }\end{array}$ & $\begin{array}{l}\text { Investigação sobre as } \\
\text { características dos vínculos } \\
\text { afetivos entre adolescentes com } \\
\text { indicadores de organização de } \\
\text { TPB e seus pais. }\end{array}$ \\
\hline
\end{tabular}


Conclusão

Quadro 1. Apresentação da síntese dos trabalhos incluídos na revisão integrativa.

\begin{tabular}{|c|c|c|c|c|}
\hline $\mathbf{N}^{\mathbf{0}}$ & Título & $\begin{array}{c}\text { Periódico e Base de } \\
\text { Dados }\end{array}$ & Autoria & Principal Temática \\
\hline 07 & $\begin{array}{l}\text { Perfil dos pacientes com diagnóstico } \\
\text { de transtorno de personalidade em uma } \\
\text { clínica de serviços em saúde mental }\end{array}$ & $\begin{array}{l}\text { Rev. Eletrônica } \\
\text { Saúde Mental Alcool } \\
\text { Drog } \\
\text { LILACS }\end{array}$ & $\begin{array}{l}\text { Reis LN, Reisdorfer E, } \\
\text { Gherardi-Donato } \text { EC }^{6} \\
2013 .\end{array}$ & 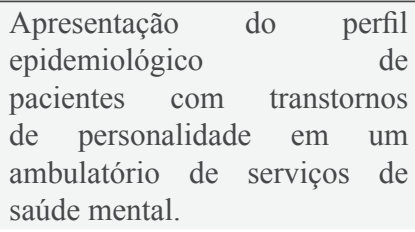 \\
\hline 08 & $\begin{array}{l}\text { Can neuroimaging be used as a support } \\
\text { to diagnosis of borderline personality } \\
\text { disorder? An approach based on } \\
\text { computational neuroanatomy and } \\
\text { machine learning }\end{array}$ & MEDLINE & $\begin{array}{l}\text { Sato JR, Araujo GM Filho, } \\
\text { Araújo TB, Bressan RA, } \\
\text { Oliveira PP, Jackowski AP } \text { AP. }^{12} \text {. } \\
\text { 2012. }\end{array}$ & $\begin{array}{l}\text { Utilização da neuroimagem } \\
\text { como suporte diagnóstico do } \\
\text { TPB. }\end{array}$ \\
\hline 09 & $\begin{array}{l}\text { Hippocampal volume in borderline } \\
\text { personality disorder with and without } \\
\text { comorbid osttraumatic stress disorder: } \\
\text { a meta-analysis }\end{array}$ & $\begin{array}{l}\text { Eur Psiquiatria } \\
\text { MEDLINE }\end{array}$ & $\begin{array}{l}\text { Rodrigues E, Wenzel A, Ribeiro } \\
\text { MP, Quarantini LC, Miranda- } \\
\text { Scippa, Sena EP, Oliveira IR }{ }^{3} \\
2011 .\end{array}$ & $\begin{array}{l}\text { Meta-análise de estudos que } \\
\text { compararam o volume do } \\
\text { hipocampo em pacientes com } \\
\text { TPB com e sem PTSD em } \\
\text { relação aos controles saudáveis. }\end{array}$ \\
\hline 10 & $\begin{array}{l}\text { Personalidade borderline e as } \\
\text { dificuldades de tratamento }\end{array}$ & $\begin{array}{l}\text { Rev. Uningá } \\
\text { LILACS }\end{array}$ & $\begin{array}{l}\text { Rosa E, Zatti CA, Baldissera R }{ }^{14} \\
2015 .\end{array}$ & $\begin{array}{l}\text { Revisão integrativa sobre o } \\
\text { tratamento de adolescentes/ } \\
\text { adultos com diagnóstico de } \\
\text { transtorno de personalidade } \\
\text { borderline. }\end{array}$ \\
\hline 11 & $\begin{array}{l}\text { A emergência do transtorno de } \\
\text { personalidade borderline: uma visão } \\
\text { comportamental }\end{array}$ & $\begin{array}{c}\text { Interação Psicologia } \\
\text { LILACS }\end{array}$ & $\begin{array}{l}\text { Sousa AC, Vandenberghe } \mathrm{L}^{16} \\
2005 .\end{array}$ & $\begin{array}{l}\text { Produto de contextos sociais } \\
\text { complexos que geraram } \\
\text { mudanças na teoria e prática } \\
\text { clínica. }\end{array}$ \\
\hline 12 & $\begin{array}{l}\text { Um caso de transtorno de personalidade } \\
\text { borderline atendido em psicoterapia } \\
\text { dinâmica breve }\end{array}$ & $\begin{array}{l}\text { Psicol. teor. Pesqui. } \\
\text { LILACS }\end{array}$ & $\begin{array}{c}\text { Cunha PJ, Azevedo MA }{ }^{17} \\
2001 .\end{array}$ & $\begin{array}{l}\text { Análise dos benefícios da PDB } \\
\text { em um caso de transtorno de } \\
\text { personalidade borderline. }\end{array}$ \\
\hline 13 & $\begin{array}{l}\text { Possibilidades da FAP como método de } \\
\text { supervisão de terapeutas com clientes } \\
\text { borderlines }\end{array}$ & $\begin{array}{l}\text { Rev. bras. ter. } \\
\text { comport. Cogn. } \\
\text { LILACS }\end{array}$ & $\begin{array}{c}\text { Sousa AC, Vandenberghe } \mathrm{L}^{7} \\
2007 .\end{array}$ & $\begin{array}{l}\text { Análise das possibilidades } \\
\text { de aplicação da FAP como } \\
\text { metodologia de supervisão em } \\
\text { caso de atendimentos a pessoas } \\
\text { com TPB. }\end{array}$ \\
\hline 14 & $\begin{array}{l}\text { Programa de abordagem interdisciplinar } \\
\text { no tratamento do transtorno de } \\
\text { personalidade borderline: relato da } \\
\text { experiência no ambulatório Melanie } \\
\text { Klein do Hospital Psiquiátrico São } \\
\text { Pedro }\end{array}$ & $\begin{array}{l}\text { Rev. psiquiatr. Rio } \\
\text { Gd. Sul } \\
\text { LILACS }\end{array}$ & $\begin{array}{l}\text { Dal'pizol A, Lima LD, Ferreira } \\
\text { LM,Martins MC, Corrêa PO, } \\
\text { Alves M, et } \mathrm{al}^{3} \\
\qquad 2003 \text {. }\end{array}$ & $\begin{array}{l}\text { Análise crítica e descritiva da } \\
\text { experiência obtida pelos autores } \\
\text { na implantação de um Programa } \\
\text { de Abordagem Interdisciplinar } \\
\text { no Tratamento do TPB. }\end{array}$ \\
\hline 15 & $\begin{array}{l}\text { Adesão ao tratamento clínico no } \\
\text { transtorno de personalidade borderline }\end{array}$ & $\begin{array}{l}\text { Estud. psicol. } \\
\text { (Natal). } \\
\text { LILA CS }\end{array}$ & $\begin{array}{l}\text { Tanesi PH, Yazigi L, Fiore ML, } \\
\text { Pitta JC }{ }^{18} \\
2007 .\end{array}$ & $\begin{array}{l}\text { Análise qualitativa do nível } \\
\text { de adesão dos pacientes ao } \\
\text { tratamento clínico do TPB. }\end{array}$ \\
\hline
\end{tabular}

Fonte: elaborado pelos autores.

Em análise aos achados desta pesquisa, apreendem-se artigos que se enquadraram nos critérios de inclusão em duas bases de dados, quais sejam: MEDLINE E LILACS.
Um desses artigos, publicado em 2014, apareceu repetido nas duas bases. Os outros, por sua vez, seguiram a seguinte divisão: na MEDLINE foram encontrados 3 artigos, destes, 
1 foi publicado em 2011 e 2 em 2012. Na LILACS, por conseguinte, estão incluídos os outros 11 artigos, dos quais 1 foi publicado em 2001; 1 em 2003; 1 em 2005; 2 em 2007; 3 em 2010; 1 em 2011; 1 em 2013 e 1 em 2015. Desse modo, observa-se que há uma distribuição equitativa da produção científica desse tema nos últimos anos e, apesar de em pequena quantidade, todos os últimos 5 anos apresentaram publicação, o que indica a existência de pesquisadores brasileiros interessados no assunto.

Quanto à origem das pesquisas, foram selecionadas apenas produções que apresentaram o Brasil como país de filiação, na busca por apreender pesquisas desenvolvidas no país. No entanto, os periódicos nos quais os trabalhos foram publicados não se restringem à circulação no Brasil. Dos selecionados, todos foram publicados em bases internacionais.

Quanto aos assuntos principais desses periódicos, salientam-se: Psiquiatria, Psicologia, Terapêutica, Neurologia, Transtornos Mentais, Ciências do Comportamento, Psicofarmacologia, Medicina, Enfermagem, Cérebro, Pesquisa em Serviços de Saúde, Farmacologia e Ciência.

No que se refere aos temas dos artigos, por sua vez, identificouse que 1 traça o perfil epidemiológico; 3 fazem uma análise contextual dos comportamentos relacionados ao TPB; 4 tratam da compreensão e análise neurobiológica do TPB; e 7 apresentam análises de estratégias de tratamento e de formas de manejo clínico, propriamente dito. Dessa forma, a maior parte dos estudos $(46,6 \%)$ se refere a aspectos relacionados ao tratamento e ao manejo clínico, fundamentais para o aprimoramento dos saberes e práticas que envolvem o cuidado à pessoa que sofre com o TPB. No entanto, isso não torna os estudos epidemiológicos menos importantes, apesar de eles estarem representados em apenas $1(6,6 \%)$ dos trabalhos selecionados.

\section{DISCUSSÃO}

Os dados encontrados demonstram que o TPB apresenta grande expressividade epidemiológica e possui uma complexidade que vai desde os seus sintomas diagnósticos, passando pelo desenvolvimento comportamental nos sujeitos, até os manejos clínicos utilizados pelos psiquiatras e demais profissionais da saúde mental.

Destarte, em estudo realizado por Reis, Reisdorfer e GherardiDonato, verificou-se que os transtornos de personalidade são os mais frequentes em um serviço de saúde mental. Além disso, pôde-se identificar que o mais prevalente é o diagnóstico $\mathrm{F} 60.3$, que se refere ao TPB, representando $70 \%$ dos diagnósticos. Em seguida, estão os transtornos de personalidade histriônica e os transtornos de personalidade inespecíficos. Com relação aos demais transtornos, observaram que eles têm prevalência consideravelmente menor. Destes, citam-se: F60, F60.2, F60.6, F60.7, F61, F61.0, F63.3, F64.0, F65, F68.1, F68.2. ${ }^{6}$

A pesquisa supracitada concluiu, ainda, que o TPB é mais prevalente entre mulheres, indicando, inclusive, relatos de pesquisas internacionais que discutem as diferenças na função serotonérgica entre homens e mulheres, e que estas podem estar relacionadas ao desenvolvimento do TPB.

Outras características investigadas que também apresentaram maior prevalência foram: a idade adulta e a baixa escolaridade. Destarte, os autores analisaram que o aparecimento do transtorno se dá, muitas vezes, de maneira precoce e isso torna os processos educacionais mais difíceis e, devido ao desconhecimento do TPB e dos cuidados em saúde mental, tem-se uma busca ao tratamento tardio, o que explicaria a maior quantidade de pessoas com idade superior a 40 anos na prevalência dos acompanhados pelo serviço de saúde mental. ${ }^{6}$

Além disso, os pesquisadores identificaram que 18\% dos indivíduos já necessitaram de internação hospitalar. Sobre esse processo interventivo, os autores apontam que, apesar das controvérsias que envolvem a internação psiquiátrica, ela ainda é um recurso muito utilizado e tem se mostrado indispensável nos casos de TPB, necessitando de tratamentos mais específicos e acompanhamento mais diretivo. ${ }^{6}$

Essa postura atenta que se faz necessária no acompanhamento terapêutico do sujeito com TPB vem como consequência de sua topografia, que se traduz em sentimentos de impotência e confusão. É comum que o indivíduo com TPB tenha dificuldade em fazer escolhas simples no seu cotidiano e, até mesmo, em expressar características e gostos próprios, ou seja: falar sobre quem ele é, do que gosta, quais os seus planos de vida. Desse modo, suas atitudes e pensamentos tendem a ser contraditórios e transitórios; o que torna o atendimento clínico um desafio constante. Além disso, devido a essa flexibilização constante do pensamento e das condutas, a taxa de desistência do tratamento é alta, sendo igualmente significativa a incidência de tentativas de suicídio. Assim, apesar de os comportamentos de TPB se desenvolverem de maneira singular em cada pessoa, existe uma tendência para essa instabilidade e contradição de conduta e pensamento. ${ }^{7}$

Nesta etapa, Jordão e Ramires apontam a dificuldade ainda maior quando se trata de TPB em adolescentes. Discutem que, por se tratar de um período de transição, marcado exatamente por flutuações, imprecisões e contradições, bem como por um processo de reorganização da identidade, tem-se como características comuns o comportamento impulsivo e outros aspectos que se assemelham aos descritos como critério diagnóstico do TPB ${ }^{8}$ Assim, a barreira entre o "normal" e o "patológico" se torna mais tênue nessa fase da vida do ser humano. Os autores indicam, portanto, a necessidade de cautela, tempo e atenção redobrada no acompanhamento e tratamento desses sujeitos. Afirmam que as principais distinções que se podem levar em consideração se resumem em que os adolescentes com TPB apresentam maior dificuldade em realizar as tarefas desenvolvimentais típicas dessa etapa, tais como: a construção de uma identidade, o desenvolvimento e afirmação sexual, o afastamento gradativo das referencias parentais e a superação do corpo infantil.

Em outro estudo realizado por Jordão e Ramires foi feita a análise da relação dos adolescentes diagnosticados com TPB 
e as suas figuras parentais. Os resultados de tal pesquisa apontaram para a existência de laços afetivos instáveis, em contextos de sofrimento, resultantes de maus tratos, solidão e ambivalência afetiva. ${ }^{9}$ Diante disso, analisa-se a dificuldade para a construção de uma identidade integrada e para construção e manutenção de vínculos afetivos saudáveis com outras pessoas. Esses fatores foram descritos como pujantes influenciadores do desencadeamento do TPB, tendo sido apresentados por outros pesquisadores citados pelos autores supracitados, quais sejam: BAIRD \& COLS, 2005; BARONE, 2003; BRADLEY, JENEI \& WESTEN, 2005; BRADLEY \& WESTEN, 2005; LEVY, 2005; LEVY \& COLS, 2007; MARANGA, 2002; e REICH \& ZANARINI, 2001, cujas referências podem ser encontradas no artigo de Jordão e Ramires. ${ }^{9}$

Outro ponto que se pode enfatizar do perfil epidemiológico do TPB é a maior frequência em mulheres. Em análise a essa característica, Sousa e Vandenberghe (2005) discutem que as mudanças que aconteceram no decorrer da história da humanidade podem ser causa importante dessa dimensão. Assim, compreende-se que, no passado, as mulheres seguiam um padrão de conduta muito fechado, que se resumia em: se casar, conceber filhos, cuidar destes, do marido e dos afazeres domésticos. Hoje, além de assumir todos estes papéis, ainda batalham com funções no mercado de trabalho fora de casa. Diante disso, elas são levadas a, muitas vezes, tomar decisões difíceis sobre a priorização de atividades familiares e do trabalho, o que, por si, já simboliza confusão e estresse. Além disso, por assumirem diferentes papéis, pode-se ocorrer a dificuldade de saber sobre quem são ou o que querem. Os homens, por sua vez, passaram por poucos ajustes, apesar das mudanças socioculturais que decorreram na história, não sofrem tanta pressão como as mulheres e nem têm que desempenhar tantos papéis. ${ }^{7}$

Por outro lado, ressalta-se que, embora as pesquisas geralmente relacionem o desenvolvimento do TPB com contextos específicos e fatores na história de vida do indivíduo, há estudos sobre a predisposição biológica, dos quais se citam os seguintes: "Alterações na morfologia cortical em mulheres com transtorno de personalidade borderline: uma abordagem multimodal". ${ }^{10}$ "O volume da amígdala em transtorno de personalidade borderline com e sem comorbidade de transtorno de estresse pós-traumático: a meta-análise"; 11 "Neuroimagem pode ser usado como um suporte para o diagnóstico de transtorno de Personalidade borderline? Uma abordagem baseada na neuroanatomia computacional e aprendizado de máquina"12 e, por último, "Volume do hipocampo em transtorno de personalidade borderline com e sem transtorno de estresse pós-traumático comorbidade: a meta-análise". ${ }^{13}$

Em meta-análise realizada por Almeida, ${ }^{11}$ por sua vez, sugeriu-se que o volume da amígdala se apresenta reduzido em indivíduos diagnosticados com TPB, contudo ainda não se pôde traçar uma conclusão exata. É preciso que se desenvolvam novos estudos - ainda mais sensíveis e determinantes - que discutam tal possibilidade com maior acurácia.
Rodrigues, ${ }^{13}$ por outro lado, fez uma meta-análise e encontrou resultados concisos que sugerem que os volumes do hipocampo são reduzidos em indivíduos com TPB, quando comparados aos controles saudáveis, sendo que este dado é ainda mais significativo nos casos em que os sujeitos são diagnosticados com a comorbidade Transtorno de Estresse Pós-Traumático.

Na pesquisa realizada por Araujo, ${ }^{10}$ por sua vez, se observou alterações morfológicas corticais significativas em mulheres com TPB, em comparação com controles saudáveis pareados por sexo. Esse achado tem a pujança de servir de base a novas e importantes investigações de neuroimagem, pois, além de apontar a existência de variação significativa na morfologia, esses pesquisadores indicaram a utilização de um novo método de análise estrutural que oferece descrições mais detalhadas sobre as características volumétricas e geométricas da superfície cortical, traçando um modelo de análise mais específico.

Sato et al, ${ }^{12}$ também realizaram investigações em alterações morfológicas cerebrais afim de encontrar possíveis associações com o TPB. Para isso, eles se basearam em estudos que compararam a concentração de substância cinzenta média entre os grupos de pessoas diagnosticadas com TPB em comparação com controles saudáveis. Além disso, consideraram as seguintes regiões do cérebro: orbitofrontal, cingulado anterior rostral, cingulado posterior, córtex temporal médio, que são as que estão distintamente envolvidas no controle emocional e participam ativamente da sintomatologia do TPB. A estratégia de investigação adotada por eles sugere grande valor clínico, na medida em que pode contribuir na construção do diagnóstico, bem como apresenta potencial para estudos de neuroimagem de outros transtornos psiquiátricos. $\mathrm{O}$ método utilizado foi uma combinação de ferramentas automatizadas-morfométricas de espessura cortical/estimativa volumétrica regional e o Support Vector classificador Machine. Indicando, assim, uma metodologia de referência para outros pesquisadores.

Compreende-se, assim, que o TPB é um transtorno de difícil diagnóstico, seja no que se refere aos aspectos comportamentais, seja em investigações de neuroimagem. Desse modo, muitas vezes, é diagnosticado tardiamente e não raramente é confundido com outros transtornos. Além disso, enfatiza-se que sua gravidade está relacionada não apenas com o risco de suicído, mas também devido à presença de comodidades. ${ }^{14}$

Uma dessas comorbidades é a Bulimia, que por envolver impulsividade e a frequência de atos auto-lesivos, bem como vômitos auto-induzidos, interferem substancialmente no prognóstico do TPB. O estudo realizado por Rosa e Santos, ${ }^{15}$ no entanto, mostrou que, apesar das dificuldades inerentes a essa situação, é possível, por meio de uma conduta atenta e responsável, alcançar resultados positivos no acompanhamento clínico. Para isso, foi importante a construção de um ambiente de confiança e acolhimento, na medida em que se pôde observar o compartilhamento de aspectos pessoais dos indivíduos, tão necessários para o bom andamento clínico. ${ }^{15}$ 
Assim sendo, para que se dê o estabelecimento de um clima favorável ao bom andamento do atendimento clínico é imprescindível que o profissional que atende pessoas com TPB tenha algum tipo de suporte, pois os comportamentos do sujeito com TPB são capazes de desestruturar o terapeuta, tornando-o contraprodutivo na sua atuação. Orienta-se, portanto, que esses profissionais busquem ser supervisionados (ou até mesmo discutir os casos com outros profissionais), para que se torne mais fácil identificar as relações que estão sendo contraprodutivas no tratamento. ${ }^{16}$

No que se refere às psicoterapias utilizadas no acompanhamento de casos de TPB, foi encontrado um estudo de Cunha e Azevedo ${ }^{17}$ em que eles reforçam a idéia de que um modelo de terapia que envolva condutas mais ativas do terapeuta, com técnicas de intervenções mais diretivas, mostra-se eficiente. Descrevem um caso bem-sucedido em que foi utilizada a Psicoterapia Dinâmica Breve e demonstram que a postura ativa do terapeuta - indicando e clarificando ao indivíduo os sintomas e os momentos em que surgiam -, possibilitou ao paciente apreender uma maior conscientização acerca do transtorno e a encontrar condições de reintegração cognitiva e emocional, melhorando, assim, a sua convivência com os sintomas.

Ressalta-se, no entanto, que essa postura ativa nem sempre vai apresentar resultados satisfatórios, depende, também, do sujeito que busca o tratamento. Às vezes, por exemplo, a pessoa precisa se aproximar de reflexões que envolvam vivências mais primitivas e, nesse caso, esse tipo de abordagem não se adequaria. Além disso, é importante frisar que a terapia farmacológica é fundamental em muitos casos. Desse modo, o tratamento mais acertado é aquele que combina a terapia que mais se adequa ao sujeito com os psicofármacos necessários - direcionados, principalmente, a sintomas-alvo específicos. ${ }^{17}$

Entende-se, então, que o manejo clínico deve, mais do que seguir uma conduta técnica, desenvolver-se de acordo com o contexto e as peculiaridades apresentadas pelo paciente.

Assim sendo, sugere-se uma conduta clínica que se inspire nas psicoterapias de orientação psicanalítica, na medida em que os processos interpretativos facilitados por essa abordagem contribuiriam satisfatoriamente para a compreensão mais acurada do TPB. Ao mesmo tempo, aponta-se a importância sobre o tema da transferência - presente na teoria psicanalista - no manejo clínico de acompanhamento do TPB. ${ }^{2}$

Nessa seara, surge, enquanto estratégia que deve ser reforçada, a integração multiprofissional. Analisa-se que por meio de escutas e olhares múltiplos é mais possível que se estabeleçam atendimentos resolutivos, na medida em que se passa a ter uma atenção integral, que valoriza os diferentes aspectos do sujeito.

De acordo com essa proposta Dal'pizol et al, ${ }^{3}$ apresentam um programa interdisciplinar e o avaliam positivamente no que se refere ao relacionamento interpessoal, à valorização dos interesses ocupacionais, de lazer e, principalmente, da autonomia dos sujeitos. Além disso, apontam que houve uma diminuição no número de internações e nas tentativas de suicídio, bem como na procura por serviços de emergência. Indicaram, assim, que um programa que reúne diferentes profissionais foi eficaz no acompanhamento de pessoas com TPB, na medida em que incrementou às terapêuticas já tradicionalmente colocadas um aspecto fundamental, que é o olhar holístico e o entendimento integralizador para o paciente.

Essa ênfase em se buscar estratégias mais eficientes no tratamento e manejo clínico de casos de TPB existe, portanto, em consonância com os desafios impostos pela própria sintomatologia do transtorno, qual seja: impulsividade, o uso de manipulações, a presença de dissociação afetiva, o risco e a ocorrência de suicídios, além das auto-agressões que se cronificam e a agressividade que está presente em quase todo o processo. Tudo isso coloca a equipe em uma situação de impotência e acaba por acontecer muitas desistências do tratamento. ${ }^{18}$

Como estratégia pujante de contornar esse quadro e aumentar a participação dos pacientes com TPB no tratamento, recomenda-se a valorização e o incentivo da participação da família, indicando-se que a presença de familiares saudáveis contribui para impulsionar os pacientes a permanecerem no tratamento e, assim, poderem desenvolver relações sociais mais saudáveis e satisfatórias. Compreende-se, portanto, que o apoio social proporcionado pela família é essencial para o bom andamento do tratamento. ${ }^{18}$

Observa-se, dessa maneira, que o psiquiatra - assim como outros profissionais de saúde -, ao se deparar com um caso de TPB, precisa estar atento aos detalhes diagnósticos e, mais do que isso, desenvolver uma postura clínica atenta e que facilite a permanência do paciente no tratamento, principalmente devido ao risco de suicídio. Avalia-se, ainda, que o essencial é aliar o acompanhamento psicoterapêutico com o tratamento farmacológico.

\section{CONSIDERAÇÕES FINAIS}

Para uma assistência adequada ao sujeito com TPB se faz necessário aliar a prática clínica às pesquisas científicas, que trazem inovações tanto no que se refere aos aspectos diagnósticos, quanto aos manejos clínico-terapêuticos. Desse modo, a partir desta revisão integrativa foi verificada a necessidade de novas investigações acerca da temática abordada, como por exemplo, sobre a acurácia diagnóstica, inclusive, como potencialidade para o tratamento do transtorno ainda em fase inicial; e não tardiamente, conforme as pesquisas apontam ser realidade.

Discorre-se sobre a complexidade que envolve o transtorno, principalmente no que se refere aos critérios diagnósticos e às graves repercussões que ele carrega, tais como autolesões e tentativas de suicídio, o que torna a emergência em se estudar e pesquisar na área.

Aponta-se a lacuna de estudos que discutam e avaliem a utilização de fármacos, principalmente porque o tratamento farmacológico foi citado como imprescindível em muitos 
estudos, essencialmente no tocante a sintomas específicos. Avalia-se que não existe uma conduta terapêutica farmacológica concisa na literatura sobre o tratamento de TPB, o que torna o trabalho do psiquiatra ainda mais desafiador.

Por fim, enfatiza-se a necessidade de que se construa uma atenção integral e atenta aos pacientes com TPB, envolvendo

\section{REFERÊNCIAS}

1. Associação Americana de Psiquiatria. Manual de diagnóstico e estatística de distúrbios mentais: DSM 5. 5.ed. Porto Alegre: Artmed; 2013.

2. Vidal M, Lowenkron T. Ensino da psicoterapia no atendimento psiquiátrico dos pacientes com transtorno de personalidade borderline. Psic: Teor e Pesq. 2010;26(4):725-8.

3. Dal'pizol A, Lima LD, Ferreira LM, Martins MC, Corrêa PO, Alves M, et al. Programa de abordagem interdisciplinar no tratamento do transtorno de personalidade borderline: relato da experiência no ambulatório Melanie Klein do Hospital Psiquiátrico São Pedro. R Psiquiatr RS. 2003;25 Supl 1:42-51.

4. Araújo AC, Lotufo F Neto. A nova classificação americana para os transtornos mentais: o DSM-5. Rev Bras de Ter Comp Cogn. 2014;21(1):67-82.

5. Mendes KD, Silveira RC, Galvão CM. Revisão integrativa: método de pesquisa para a incorporação de evidências na saúde e na enfermagem. Texto Contexto Enferm. 2008;17(4):758-64.

6. Reis LN, Reisdorfer E, Gherardi-Donato EC. Perfil dos usuários com diagnóstico de transtornos de personalidade de um serviço de saúde mental. Rev Eletrônica Saúde Mental Álcool Drog. 2013;9(2):70-5.

7. Sousa AC, Vandenberghe L. A emergência do transtorno de personalidade borderline: uma visão comportamental. Interação em Psicologia. 2005;9(2):381-90.

8. Jordão $A B$, Ramires VR. Adolescência e organização de personalidade borderline: caracterização dos vínculos afetivos. Paidéia. 2010a; 20(47):421-30.

9. Jordão $A B$, Ramires VR. Vínculos afetivos de adolescentes borderline e seus pais. Rev. Psic: Teor e Pesq. 2010b;26(1):89-98.

10. Araujo TB, Araújo GM Filho, Sato JR, Araújo CM, Lisondo seus familiares e considerando seus contextos vivenciais, na busca por produzir um cuidado resolutivo, diminuindo o sofrimento destas pessoas. Recomenda-se, ainda, a existência de supervisões e/ou discussão de casos, na medida em que esse transtorno apresenta peculiaridades significativas, como a agressividade e a impulsividade.

CM, Carret H Júnior, et al. Alterações na morfologia Cortical muda em mulheres com transtorno de personalidade borderline: uma abordagem multimodal. Rev Bras Psiquiatr. 2014;36(1):32-8.

11. Almeida CP, Wenzel A, Carvalho CS, Powell VB, Araújo C Neto, Quarantini LC, et al. Amygdalar volume in borderline personality disorder with and without comorbid post-traumatic stress disorder: a meta-analysis. Rev CNS Spectr. 2012;17(2):70-5.

12. Sato JR, Araujo GM Filho, Araújo TB, Bressan RA, Oliveira PP, Jackowski AP. Can neuroimaging be used as a support to diagnosis of borderline personality disorder? An approach based on computational neuroanatomy and machine learning. Rev J Psychiatr Res. 2012;46(9):1126-32.

13. Rodrigues E, Wenzel A, Ribeiro MP, Quarantini LC, MirandaScippa, Sena EP, et al. Hippocampal volume in borderline personality disorder with and without comorbid osttraumatic stress disorder: a meta-analysis. Rev Eur Psychiatry. 2011;26(7):452-6.

14. Rosa E, Zatti CA, Baldissera R. Personalidade borderline e as dificuldades de tratamento. Rev Uningá. 2015;21(1):5-10.

15. Rosa BP, Santos MA. Comorbidade Entre bulimia e transtorno de personalidade limítrofe: implicações para o tratamento. Rev latinoam psicopat fund. 2011;14(2):268-82.

16. Sousa AC, Vandenberghe L. Possibilidades da FAP como método de supervisão de terapeutas com clientes boderlines. Rev Bras de Ter Comp Cogn. 2007;9(1):1-11.

17. Cunha PJ, Azevedo MA. Um caso de transtorno de personalidade borderline atendido em psicoterapia dinâmica breve. Psic: Teor. e Pesq. 2001;17(1):5-11.

18. Tanesi PH, Yazigi L, Fiore ML, Pitta JC. Adesão Ao tratamento clínico no transtorno de personalidade borderline. Estud Psicol (Natal). 2007;12(1):71-8.

\section{Como citar:}

Leite LH, Campos EM. Investigações brasileiras sobre o transtorno de personalidade borderline: uma revisão integrativa. Rev Med UFC. 2016 jan-jun;56(1):29-37. 